\title{
Zur lettischen Literatur in der Sowjetzeit: Strategien des Widerstandes aus postkolonialer Perspektive
}

\author{
BENEDIKTS KALNAČS
}

\begin{abstract}
The article provides an interpretation of the development of Latvian literature during the Soviet period. In order to grasp historical connections, an overview of the literary, cultural and social development in the territory of the present day Latvia in a comparative perspective is attempted as well. The historical processes in Latvian literature are dealt with against the background of the colonial experience from the beginning of the Latvian letters in the $16^{\text {th }}$ century onwards. The literary development during the Soviet era is seen as a repetition of an earlier pattern of colonial experience with foreign models gradually being appropriated and transformed in the creative output of representatives of the local cultural elite. To describe the cultural logic of Soviet colonialism and its gradual overturn, postcolonial critical methodology is used as a research tool and its applicability to Latvian literature discussed in the context of contemporary scholarly discourse. Investigations of Soviet colonialism undertaken by researchers of Baltic literatures and cultures (Violeta Kelertas, Epp Annus) as well as East-Central European researchers (Cristina Șandru, Andrei Terian, Madina Tlostanova, Nataša Kovačeviḉ) are involved in the discussion alongside various perspectives upon global colonial relations (Edward Said, Bill Ashcroft, Walter D. Mignolo, Jürgen Osterhammel et al.). The impact of Soviet ideology and colonial presence upon Latvian literature is discussed while providing a three-step pattern in the development of literature of the Soviet period. The early phase of the Soviet ideological presence (till approximately the mid-1950s) with its emphasis on a complete renewal of literary process is followed by two periods where critical appropriation and inversion of the Soviet model become dominant. The final part of the article provides a case study of the work of the Latvian dramatist Gunārs Priede (1928-2000) whose formative years reveal the impact of the Soviet ideological pressure while, on the other hand, Priede's dramaturgical output from the mid-1950s onwards initiates new trends which are marked by latent manifestation of anti-colonial self-consciousness of the Latvian nation.
\end{abstract}

Keywords: postcolonial criticism, Soviet colonialism, Latvian literature, Gunārs Priede

DOI: http://dx.doi.org/10.12697/IL.2013.18.2.18 
Der vorliegende Aufsatz skizziert die literarische Entwicklung auf dem Territorium des heutigen Lettlands aus postkolonialer Perspektive. Der Schwerpunkt der Analyse liegt auf der zweiten Hälfte des 20. Jahrhunderts, als die sowjetische Herrschaft die Doktrin des Sozialistischen Realismus zu erzwingen versuchte. Diese Phase wird allerdings vor einem größeren Hintergrund betrachtet, insofern die lettische Literatur der Sowjetzeit im Kontext der Entwicklungsmöglichkeiten der lettischen Literatur generell betrachtet wird. Denn koloniale Zustände verbinden verschiedene Phasen der Literaturentwicklung. Daher werde ich zunächst die lettische Literaturgeschichte kurz umreißen, denn diese weist aufschlussreiche Parallelen zu den Tendenzen des 20. Jahrhunderts, jedoch in einem größeren Zeitraum, auf. Danach folgt ein Forschungsüberblick über die so genannte postkoloniale Kritik in Hinblick auf das Baltikum. Die weitere Entwicklung der lettischen Literatur in der zweiten Hälfte des 20. Jahrhunderts analysiere ich sodann mithilfe der postkolonialen Begrifflichkeit. Der Aufsatz schließt mit einer Fallstudie über die literarische Tätigkeit des Dramatikers Gunārs Priede.

\section{Entwicklung der lettischen Literatur. Versuch einer Periodisierung}

Bis ins frühe 20. Jahrhundert kann man die Entwicklung der lettischen Literatur in drei Stufen einteilen. Das Schreiben auf Lettisch (sowie auf Estnisch) begann im 16. Jahrhundert in Texten, die von deutschen Pastoren verfasst wurden. Diese Texte, die inhaltlich zunächst religiös, später auch weltlich waren und von den Intellektuellen der damaligen Zeit produziert wurden, beinhalten eine bestimmte soziale Perspektive, die die Machtverhältnisse in der Gesellschaft eher stärkte, als dass sie diese selbst infragestellte. Im Laufe des 19. Jahrhunderts verändert sich diese Perspektive langsam, aber eher im politischen als im ästhetischen Sinne. Die damalige estnische (und gleichfalls die lettische) Literatur war, wie Liina Lukas es beschreibt, „eher eine Fortsetzung der deutschen Literatur in estnischer (oder auch lettischer) Sprache." (Lukas 2006: 78) Das heißt auch, dass die früheren literarischen Modelle bewahrt wurden. Hinzu kam eine starke zaristische Zensur, insbesondere was die Texte auf Lettisch oder Estnisch betraf.

Innerhalb dieser Prozesse machte sich graduell auch eine andere Tendenz bemerkbar, die bestimmt war von ideologisch motivierten Bestrebungen der jungen lettischen - sowie auch estnischen - Autoren, die versuchten, eine Änderung der Machtverhältnisse zwischen dem deutschen (und bzw. auch dem russischen) Teil der Bevölkerung einerseits und dem lettischen bzw. estnischen 
KALNAČS

Volk andererseits herbeizuführen. Ästhetisch blieben diese Texte in vielen Fällen der deutschen Tradition verhaftet. Gleichzeitig aber wurden die Weichen neu gestellt, und das Widerstandspotenzial solcher literarischen Werke wurde gleich von beiden Seiten erkannt.

Eine dritte Entwicklungsetappe lässt sich auf die Jahrhundertwende datieren, und sie wurde von dem Bestreben bestimmt, sich nicht nur den lokalen Traditionen der deutsch-baltischen Autoren anzupassen, sondern die gesamte Tradition der Weltliteratur so rasch wie möglich zu rezipieren und, durch diese Erfahrung bereichert, selbstständige und innovative Texte zu verfassen, die den modernsten Tendenzen der Zeit entsprachen und auch die geistige Selbstständigkeit des aufstrebenden Volkes bestätigten. Diese Zielrichtung wurde auch in den 1920er und 1930er Jahren bewahrt, auch wenn die früher oft provozierenden jungen Intellektuellen jetzt stärkere äußere Solidität zeigten und die konservativen Regierungen der unabhängigen Staaten in den 1930er Jahren nicht ohne Einfluss auf die Bewahrung der literarischen Tradition waren.

\section{Die Sowjetzeit im Baltikum: zwischen Okkupation und Kolonialismus}

Seit einigen Jahren hat sich die literaturwissenschaftliche und historische Forschung in Estland und Lettland die Frage vorgelegt, ob sich die Geschichte des Baltikums einschließlich der sowjetischen Erfahrung mit Begriffen beschreiben lässt, die von der so genannten postkolonialen Kritik aufgearbeitet wurden. Oder anders formuliert: Wie und auf welche Weise ist die Verwendung von Begriffen wie Kolonialismus und Postkolonalismus in Bezug auf die lettische (und estnische) Literatur möglich?

Kolonialismus soll im Folgenden als eine Verwendung der Macht verstanden werden, die sich in der Besetzung eines fremden Territoriums und in der damit verbundenen Neubesiedlung durch die Eroberer manifestiert, wobei die zuvor dominierenden sozialen und kulturellen Verhältnisse vernichtet und neu definiert werden. In der Kolonialismusforschung wurde bereits wiederholt nachgewiesen, dass die Eroberung Amerikas im späten 15. Jahrhundert nicht nur die europäische Vormachtstellung zur Folge hatte, sondern die europäische Identität allererst konstruiert hat (vgl. etwa Mignolo 2011). Diese ideologische Entwicklung vollzieht sich spätestens im 18. Jahrhundert, als insbesondere Großmächte wie England und Frankreich als Musterbeispiele für den menschlichen Fortschritt angesehen wurden. Im 19. und 20. Jahrhundert durchläuft das koloniale System weitere Veränderungen, und die Versuche einer neuen territorialen Machtaufteilung provozieren globale Konflikte, inklusive 
die beiden Weltkriege. Andererseits ist auch die Entwicklung antikolonialer Bewegungen $\mathrm{zu}$ beobachten, die sowohl politisch als auch theoretisch Veränderungen im weltweiten Machtgefüge widerspiegeln (vgl. hierzu z. B. die Arbeiten Frantz Fanons). In vielen Publikationen wird auch der Begriff des kolonialen Diskurses diskutiert, wobei dieser Begriff auf ein ideologisch umfassendes Netz von Vorstellungen fokussiert, mit denen koloniale Mächte ihre Herrschaft umsetzen (Said 1978). In engem Zusammenhang damit steht der Begriff der postkolonialen Literatur, der sich auf Texte bezieht, die in Zeiten kolonialer Herrschaft sowie auch nach deren Ende verfasst wurden und deren Autoren einer kolonial unterdrückten Gesellschaft entstammen (vgl. Ashroft et al. 1989).

Wenn man diese Entwicklungen im Kontext der heutigen Länder des Baltikums näher betrachtet, so gibt es bei allen Differenzen im Detail eine historisch vergleichbare Entwicklungstendenz. Die Entdeckung dieser Länder und die Besetzung ihres nördlichen Teils im 12. und 13. Jahrhundert unter der Ideologie der christlichen Seefahrt etablierte neue Machtverhältnisse, und dies wiederum spielte eine Rolle für die Aufteilung der Welt, die in späteren Jahrhunderten weiter stabilisiert wurde. Die späteren europäischen Eroberungen seit dem 16. Jahrhundert geschahen zeitgleich mit dem Vormarsch des Russischen Imperiums (Kappeler 1992), und seine weitere territoriale Expansion im 18. Jahrhundert markierte auch die politische Dominanz Russlands im Baltikum.

Aus diesem Blickwinkel stellen sich die Unabhängigkeitsbestrebungen im 19. und 20. Jahrhundert in den baltischen Ländern als Teil einer globalen antikolonialen Bewegung dar und die Geschichte dieser Länder als Teil größerer weltpolitischer Entwicklungen.

Die Tatsache, dass die sowjetische Politik für so lange Zeit nicht mit dem Begriff Kolonialismus in Verbindung gebracht wurde, ist dadurch zu erklären, dass die postkoloniale Perspektive auch im Westen als eine Form innerer Kritik gesehen wurde (vgl. Annus 2012: 24). Der Versuch, einem sozialistischen Staat wie der Sowjetunion Parallelen mit der kapitalistischen Welt nachzuweisen, war damals völlig undenkbar. Doch die sowjetische Realität war in zentralen Aspekten durchaus mit der Kolonialgeschichte und der daraus resultierenden Machtverhältnisse vergleichbar.

Die sowjetische Besetzung des Baltikums nach dem Zweiten Weltkrieg wird meist unter dem Begriff „Okkupation” zusammengefasst. Doch, wie etwa die Studien von Epp Annus belegen, folgen auf diese Okkupation weitere Schritte, die auf die politische, ökonomische und ideologische Herrschaft des sowjetischen Staates zielen und den generellen Tendenzen einer Kolonisation entsprechen (Annus 2012; Annus 2011). 
KALNAČS

In der zweiten Hälfte des 20. Jahrhunderts ist sogar eine Eskalation des Kampfes zwischen zwei verschiedenen ideologischen und politischen Systemen zu beobachten, die eine neue Stufe der Weltteilung markiert. Dieser Kampf war eng mit dem sogenannten Kalten Krieg verbunden, der als Fortsetzung der kolonialen Politik früherer Prägung verstanden werden kann. In einem Artikel, der am 31. Oktober 2012 in der Frankfurter Allgemeinen Zeitung (FAZ) zu lesen war, schildert der deutsche Historiker Jürgen Osterhammel die politischen Tendenzen im dritten Viertel des 20. Jahrhunderts mit Formulierungen, die in diese Richtung weisen:

Der kalte Krieg war die Fortsetzung des Imperialismus mit anderen Mitteln, freilich eines Imperialismus, der stärker für eigene Fehler anfällig war, als es seine britischen und französischen Vorgänger im 19. Jahrhundert gewesen waren. Er endete nicht mit einem eindeutigen Sieg des Westens. [...] Überhaupt und das wäre der Kern einer globalhistorischen Neuinterpretation des Kalten Krieges - waren Asien, Afrika und Lateinamerika nicht bloß Nebenschauplätze einer Nordatlantischen ,Systemkonkurrenz', sondern von Anfang an zentrale Arenen weltpolitischer Konflikte, die von der Supermächten niemals beherrscht werden konnten. (Osterhammel 2012: 6)

Erst diese globale Sichtweise erlaubt es, die sozialpsychologischen und psychischen Prozesse in einem kolonialen Staat zu begreifen, der mit aller Macht versuchte, sich eine exklusive Dominanz zu verschaffen und in außenpolitischen Auseinandersetzungen stets sein wahres Gesicht des Eroberers öffentlich zeigte, obwohl offiziell die Rede immer nur von einem globalen Frieden (unter dem Banner der sowjetischen Ideologie) war.

In einem 2001 veröffentlichten Aufsatz hat David Chione Moore als erster die Beziehung zwischen Postkommunismus und Postkolonialismus in einer globalen Perspektive angedeutet (Moore 2006). Auch die erste von Historikern aus dem Baltikum über das Kolonialismus-Thema veröffentlichte Publikation richtet ihr Hauptaugenmerk auf die Sowjetzeit (vgl. Kelertas 2006). Mittlerweile ist eine heftige Diskussion über die Zulässigkeit dieses Begriffs für den mitteleuropäischen und mittelosteuropäischen Raum entbrannt (vgl. Şandru 2012; Terian 2012; Kovačević 2008). Auch die verschiedenen Facetten der russischen sowie der späteren sowjetischen Kolonialmacht stehen im Zentrum aktueller Forschung (Tlostanova 2012; Thompson 2000). 


\section{Die lettische Literatur in der Sowjetzeit: Versuche des Wider- stands gegen eine koloniale Herrschaft}

Ein wichtiges Ziel eines jeden Machthabers in kolonialen Zusammenhängen ist es, die Selbstständigkeit der kolonisierten Gesellschaft in Frage zu stellen und deren Vorstellungen nach Möglichkeit durch das eigene Weltbild zu ersetzen. Wenn man aus einer solchen Perspektive den Blick auf die lettische Literatur der Sowjetzeit sowie die Entwicklungen nach ihrem Ende wirft, ist eine Dynamik zu beobachten, die den Prozessen in früheren Perioden der Literaturentwicklung recht ähnlich ist.

Die lettische Literatur nach 1940 wird zunächst ganz und gar von fremden Vorstellungen und Modellen bestimmt. Die sowjetische Machtund Kolonialpolitik nach 1940 war bestrebt, im Baltikum so etwas wie einen Neubeginn zu initiieren. Alles, was mit der früheren Geschichte (inklusive der deutschen Präsenz im Baltikum) verbunden war, wurde so brutal wie möglich aus der Erinnerung getilgt. Dazu passt die buchstäbliche Vernichtung der Bücher - Prozesse, die in den Arbeiten von Heinrihs Strods (Strods 2010: 144) und Cornelius Hasselblatt (Hasselblatt 2006: 522) dokumentiert sind. Aus der Idee des Neuanfangs speist sich zumindest teilweise auch die oft unglaubliche Naivität der Literatur in der Dekade unmittelbar nach dem Zweiten Weltkrieg, gepaart mit einem von der politischen und ideologischen Linie der kommunistischen Partei ausgehenden enormen Druck auf das kreative Bewusstsein der Schriftsteller. Die Modelle, die sich in der Sowjetunion schon seit den frühen 1930er Jahren voll etabliert hatten, wurden jetzt direkt auf ein völlig anderes kulturelles Milieu übertragen. Wie das ganze Imperium so sollten auch die neu hinzugekommenen Territorien aussehen und präsentiert werden (vgl. Briedis 2010: 41). Von einer eigenständigen Entwicklung konnte keine Rede sein.

Wenn wir uns den Prozessen widmen, die sich in der lettischen Literatur während derZeit zwischen 1955 und etwa 1969 abspielten, erhalten wir zunächst ein widersprüchliches Bild. Es war ein Zeitabschnitt, in dem die literarische Produktion aufblühen konnte, aber gleichzeitig blieb man abhängig von dem, was die ästhetischen Modelle im Sinne des sowjetischen Diskurses erforderten. Das politische Diktat war in den meisten Texten wenigstens indirekt präsent; und wenn die Schriftsteller sich nicht staats- und ideologiekonform verhielten, mussten sie stets auf entsprechende Konsequenzen gefasst sein. Etwa seit Mitte der 1950er Jahre kann man jedoch gleichzeitig auch so etwas wie ein unterirdisches Brodeln vernehmen, das einen deutlichen Wandel des generellen Klimas markiert, und während der 1960er Jahre setzt sich diese Entwicklung dann fort (vgl. Kalnačs 2011: 129-147). Zu dieser Zeit gibt es 
eine anhaltende Spannung zwischen Zensur und Zeitgeist, die fast überall zu beobachten ist und die die Persönlichkeit eines jeden Dichters spaltete. Einerseits musste man eine generell parteitreue Linie verfolgen, andererseits aber versuchte man, den wechselnden Empfindungen des eigenen Volkes treu zu bleiben. Es gibt verschiedene literarische Strategien, die die Schriftsteller in den späten 1950er und 1960er Jahren nutzten, um sich irgendwie dem herrschenden Regime anzupassen und dennoch gleichzeitig Selbstständigkeit $\mathrm{zu}$ bewahren. In der lettischen sowie in der estnischen Literatur kann man zu dieser Zeit mit Berechtigung auch von einem Generationswechsel sprechen, bei dem solche Persönlichkeiten an Einfluss gewannen, deren Rolle Rein Veidemann als die 1960er-Generation zusammenfasst (Veidemann 2003: 62), die aber in den meisten Fällen schon in den 1950er Jahren ihre Karrieren begonnen hatten. Ein wichtiger Unterschied ist durch die Veränderungen im generellen politischen Klima zu erklären. Einerseits hat die kommunistische Partei auch in den späten 1950er Jahren dieselben Prinzipien bewahrt, die das gesamte Leben unter den herrschenden Bedingungen wie ein Aquarium wirken ließen, wobei fast alles, was außerhalb dessen passierte, unerreichbar blieb oder wenigstens bleiben sollte. Gleichzeitig aber änderten sich die Methoden, den erwünschten Vorstellungen der gesellschaftlichen Entwicklung mehr oder weniger nahezukommen, zumindest in dem Sinne, dass es jetzt weniger direkte Angriffe auf Literatur und Kunst gab und die Brutalität der vorherigen Dekade sich ein wenig entschärfte. Damit war auch der Eintritt einer anderen Generation in die Literatur vorbereitet, die sich wenigstens teilweise traute, ihre eigene Meinung auszusprechen.

Die dritte Phase ist eng mit dem Prozess der Enttäuschung verbunden, die insbesondere nach dem Ende des sogenannten Sozialismus mit menschlichem Antlitz in den späten 1960er Jahren einkehrt und direkt oder auch indirekt die Normen des Sozialistischen Realismus in Frage stellt. Die Prozesse, die sich in den 1960er Jahren anbahnten, mündeten später in eine neue Entwicklungsstufe der Literatur der Sowjetzeit im Baltikum. Eine Abkehr der lettischen Schriftsteller von der parteitreuen politischen Linie während der 1970er Jahre ist zum Beispiel dort klar zu erkennen, wo die früher stets verwendeten Konzepte eine gegensätzliche Deutung bekamen, wie es der Literaturkritiker Guntis Berelis im Falle der literarischen Tätigkeit von Imants Ziedonis in den 1970er Jahren beobachtet. In seiner Besprechung der Gedichtsammlung In der mir zugetanen Dunkelheit (lettisch: Man labvēlīgà tumsā, 1975) behauptet der Literaturkritiker Folgendes: 
In der lettischen Literatur ist die Dunkelheit traditionell ein Gegenteil zum Licht, ein Helfershelfer der bösen Kräfte, ein dem Chaos gewogenes Umfeld. Im Gegensatz dazu ist dieser Band der Rechtfertigung der Dunkelheit gewidmet, hier wird versucht, eine konzeptuelle Einheit zu erreichen und sich von allem Unnötigen zu befreien. Eigentlich beschäftigt sich dieses Buch mit der Aufstellung einer einzigen Metapher und ihrer poetischen Analyse. (Berelis 2005: 8)

Hinzuzufügen bleibt, dass diese Schilderung sicherlich nicht dem Positivismus sowjetischer Prägung zustimmt, sondern der Schriftsteller seinen eigenen Weg zu markieren versucht und der Kritiker ihm dabei zustimmt.

Es ist eben diese Individualisierung des literarischen Ausdruckes, die seit der zweiten Hälfte der 1950er Jahre schrittweise einkehrt, die den Ansprüchen an eine antikoloniale Dichtung entspricht und sich dann in den späteren Dekaden der sowjetischen Besetzung auch durchsetzt.

Die skizzierten Phasen sind teilweise auch parallel zur Entwicklung anderer Literaturen, die im Einflussbereich der Sowjetunion und des Sozialistischen Realismus standen. Die Besonderheit der Entwicklung der lettischen (sowie auch der estnischen) Literatur besteht jedoch darin, dass eine erneute koloniale Situation unter veränderten ideologischen Bedingungen einkehrt, wodurch die vorgeschriebenen Denkmuster einerseits von der sowjetischen Ideologie bestimmt, andererseits aber noch von der früheren Denkweise entfremdet sind, was die Möglichkeiten der Orientierung in der neu definierten Situation zusätzlich erschwert.

\section{Eine Fallstudie: der Dramatiker Gunārs Priede (1928-2000)}

Im Folgenden gebe ich einen kurzen Überblick über die literarische Tätigkeit von Gunārs Priede, die ich als Fallstudie dafür verstehe, was sich in der Sowjetzeit im literarischen Leben Lettlands abspielte. Auch bei Priede ist eine dreistufige Entwicklung des literarischen Schaffens zu beobachten.

Priede ist einer der bekanntesten lettischen Dramatiker der zweiten Hälfte des 20. Jahrhunderts. Er wurde 1928 geboren, und sein erstes Stück wurde 1955 uraufgeführt. Danach verfasste er viele weitere Dramen und wurde als Erneuerer des lettischen Dramas gefeiert. Für längere Zeit war Priede auch als Vorsitzender des lettischen Schriftstellerverbands tätig. Ungeachtet dessen fiel er wiederholt der Zensur zum Opfer.

Nach dem Abitur am Gymnasium in Ventspils (1946), wo viel Wert auf die Entwicklung der Persönlichkeit der Schüler gelegt wurde (und das in 
KALNAČS

einer Zeitspanne, wo generell die Bildungstradition aus der ersten lettischen Republik fortgesetzt wurde), kommt Priede als Architekturstudent nach Riga. In seinen Tagebüchern und Briefen der späten 1940er Jahre, die im ersten Band seiner Schriften veröffentlicht sind (Priede 2013), kann man eine Tendenz zur Häufung sowjetischer Lexik beobachten, wenngleich eine kritische Auseinandersetzung mit der offiziellen Propaganda beibehalten wird. Aber man erkennt auch die widrigen Lebensbedingungen in der damaligen sowjetischen Republik und besonders das ideologische Diktat, das sich in den Beobachtungen des jungen Autors konstant widerspiegelt. Diese emotionale Mischung ist besonders auch für die frühen Stücke Priedes sehr charakteristisch, obwohl sie zu Recht als Übergang zu einer neuen Stufe der Realitätsschilderung in der lettischen Literatur der Sowjetzeit gelten.

Sein erstes aufgeführtes Stück, Der Sommer des jüngeren Bruders (lettisch: Jaunākā brāla vasara), entstand während der ersten Hälfte der 1950er Jahre, als Priede die Anzeige eines literarischen Wettbewerbs in der Zeitung las (vgl. Priede 2013: 89). Er entschied sich, an dem Wettbewerb teilzunehmen, und schrieb seine Absichten im Notizbuch nieder, in einer Form, die eigentlich den Vorstellungen des Sozialistischen Realismus regelrecht entsprach. Im Schaffensprozess änderte sich dann vieles, und als das Stück 1955 inszeniert wurde, sah es wie ein zum damaligen Zeitpunkt neues Werk aus. Was mit großer Freude wahrgenommen wurde, war insbesondere die Authentizität der Dialoge und die intensive Schilderung der Schwierigkeiten, die auch der jüngeren Generation im Weg standen. Als aktiver Pädagoge hat Priede es verstanden, den wechselnden Emotionslagen der jungen Menschen zu folgen und damit auch der widersprüchlichen Realität auf der Spur zu bleiben.

Der so eingeschlagene Weg führte ihn weiter zu einer tiefgreifenden Kritik an den herrschenden gesellschaftlichen Verhältnissen am Beispiel des täglichen Lebens der Schulkinder, der als ein Konflikt zwischen Ideal und Wirklichkeit gefasst wird (Vikas erster Ball/Vikas pirmā balle, 1960) oder als Konflikt zwischen dem Künstler und der Institution der Zensur, deren Aktivität durch einen Bericht in Gang gesetzt wird und danach nicht mehr aufzuhalten ist (Das Dreizehnte [Stück]/ Trīspadsmitā, 1966). Gegen Ende der 1960er äußert Priede dann sogar öffentlich Zweifel an dem ideologischen Fundament, auf dem die sozialistische Gesellschaft zu basieren scheint. Dieses Stück, Die Pilze duften (Smaržo sēnes, 1967), wurde verboten und jegliche Inszenierung der Werke Priedes untersagt. Nur das energische Auftreten des Spielleiters Ādolfs Šapiro hat dann dem Schriftsteller im Jahre 1972 den Weg zurück auf die Bühne geebnet.

In seinen späteren Texten gehört Priedes Aufmerksamkeit oft dem $\mathrm{Zu}$ sammenhang von sowjetischem Alltagsleben und der Geschichte, der einen 
weiteren historischen Horizont manifestiert. Insbesondere mit dem Stück Die Zentrifuge (Centrifüga, 1984), dessen Ereignisse sich während des Zweiten Weltkriegs in Lettland abspielen, das zu diesem Zeitpunkt von den Nazis besetzt ist, gelingt dem Schriftsteller eine humane Schilderung der menschlichen Schicksale, die sich von keiner ideologischen Position oder den Wünschen der sowjetischen Herrscher beeindruckt zeigt. Die Letten, wie auch die Deutschen, die Russen, und die Weißrussen, die in diesem Stück geschildert sind, werden in der Fülle ihrer menschlichen Widersprüche beobachtet. Die hier geschilderten Schreibstrategien in der Sowjetzeit kann man unter Begriffen wie kritische Nutzung, Umgestaltung und Ablehnung zusammenfassen. Sie illustrieren den Prozess der Enttäuschung der lettischen Autoren über die zeitbedingte Ideologie und markieren die Rückkehr zur Realitätsschilderung, eine Entwicklung, die als Konsequenz den Rückzug in eine eigene Vorstellungswelt andeutet.

Man kann diese Entwicklung auch als das Bewusstwerden der kolonialen Situation beschreiben, ein Ergebnis der kritischen Aufeinandersetzung mit der Realität während der Sowjetzeit.

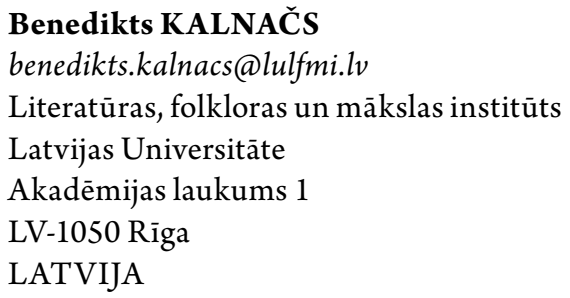

\section{Literaturverzeichnis}

Annus, E. 2012. The Problem of Soviet Colonialism in the Baltics. - Journal of Baltic Studies, 43/1, 21-46.

Annus, E. 2011. The Conditions of Soviet Colonialism. - Interlitteraria, 16/2, 441450 .

Ashroft, B., Grifftihs, G., Tiffin, H. 1989. The Empire Writes Back: Theory and Practice in Post-Colonial Literatures. London/New York: Routledge.

Berelis, G. 2005. Imants Ziedonis. - Lettische Literatur, 1, 7-9.

Briedis, R. 2010. Teksta cenzūras isais kurss: prozas teksts un cenzūra padomju gados Latvijā. Rìga: LU LFMI.

Hasselblatt, C. 2006. Geschichte der estnischen Literatur: Von den Anfängen bis zur Gegenwart. Berlin/New York: Walter de Gruyter. 
KALNAČS

Kalnačs, B. 2011. Baltijas postkoloniālā drāma: modernitāte, koloniālisms un postkoloniālisms latviešu, igauņu un lietuviešu dramaturơijā. Rīga: LU LFMI.

Kappeler, A. 1992. Russland als Vielvölkerreich: Entstehung, Geschichte, Zerfall. München: Beck.

Kelertas, V., ed. 2006. Baltic Postcolonialism. Amsterdam: Rodopi.

Kovačević, N. 2008 Narrating Post/Communism: Colonial Discourse and Europe's Borderline Civilization. London: Routledge.

Lukas, L. 2006. Die Deutschbalten auf dem estnischen literarischen Feld um 1900. -

C. Hasselblatt, ed., Different inputs - same output? Autonomy and dependence of the arts under different social-economic conditions: the Estonian example. Maastricht: Shaker, 72-88.

Mignolo, W. D. 2011. The Darker Side of Western Modernity: Global Futures, Decolonial Options. Durham/London: Duke University Press.

Moore, D. C. 2006. Is the Post- in Postcolonial the Post- in Post-Soviet? Toward a Global Postcolonial Critique. - V. Kelertas, ed., Baltic Postcolonialism. Amsterdam: Rodopi, 11-44.

Osterhammel, J. 2012. Von einem hohen Turme aus. - Frankfurter Allgemeine Zeitung, 254 (31.10.2012), 6.

Priede, G. 2013. Gunāra Priedes dzīve un darbi 1928-1958. Rīga: Jumava.

Said, E. 1978. Orientalism. New York: Pantheon Books.

Şandru, C. 2012. Worlds Apart? A Postcolonial Reading of post-1945 East-Central European Culture. Newcastle upon Tyne: Cambridge Scholars Publishing.

Strods, H. 2010. PSRS politiskā cenzūra Latvijā 1940-1990. Rīga: Jumava.

Terian, A. 2012. Is There an East-Central European Postcolonialism? Towards a Unified Theory of (Inter)Literary Dependency. - World Literature Studies, 3, 21-36.

Thompson, E. M. 2000. Imperial Knowledge: Russian Literature and Colonialism. Westport / Connecticut / London: Greenwood Press.

Tlostanova, M. 2012. Postsocialist $\neq$ Postcolonial? On Post-Soviet Imaginary and Global Coloniality. - Journal of Postcolonial Writing, 48/2, 130-142.

Veidemann, R. 2003. Literature as the factor of Social Coherence: Estonia's Case. Interlitteraria, 8, 55-67. 